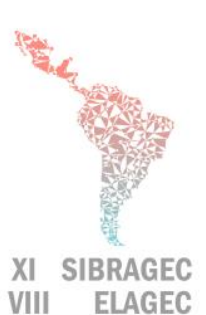

VIII ELAGEC
XI SIMPÓSIO BRASILEIRO DE GESTÃO E ECONOMIA DA CONSTRUÇÃO

VIII ENCUENTRO LATINOAMERICANO DE GESTIÓN Y ECONOMÍA DE LA CONSTRUCCIÓN

Do conhecimento à ação: práticas avançadas de gestão da produção Londrina, Paraná, Brasil. 23 a 25 de Outubro de 2019

\title{
PLANEJAMENTO DA SEGURANÇA NA CONSTRUÇÃO UTILIZANDO A MODELAGEM VIRTUAL
}

\section{LEÃO, Carla Barroso de Oliveira (1); SERRA, Sheyla Mara Baptista (2)}

(1) Doutoranda do Programa de Pós-Graduação em Engenharia Civil. Universidade Federal de São Carlos (UFSCar), cahbarroso@gmail.com (2) Departamento de Engenharia Civil, UFSCar, sheylabs@ufscar.br

\begin{abstract}
The architects and engineers can incorporate safety and health aspects of construction workers through "Prevention Trough Design" (PtD). This tool aims to integrate decisions of the safety and health at all stages of the development of the project. There is computational support for PtD with the use of virtual prototyping. The objective of this paper is to demonstrate the technical viability of integrating the planning of collective protection systems (SPC) to the schedule using the PtD (virtual prototyping) concept in a BIM$4 D$ model. The research approach was divided as follows: Definition of the chosen SPC design; Elaboration of the corresponding BIM library; Inclusion of SPC in the BIM-3D model; Planning of activities and simulation of model integrating activities related to temporary structures; Identification of opportunities for improvement in programming, especially with regard to site safety. As a result, the deficiencies were detected in programming, such as lack of guard-rails in perimeter of slabs that were being prepared for concreting. Finally, the incorporation the PtD concept is technically feasible and can help builders to anticipate risk and plan safety resource allocations in advance.
\end{abstract}

Keywords: Safety planning, Virtual prototyping, Prevention Trough Design (PtD), Building Information Modeling (BIM).

\section{INTRODUÇÃO}

Um dos setores propensos a graves acidentes de trabalho é a indústria da construção, na qual os colaboradores estão expostos a riscos e danos a saúde, provocando a hospitalização, incapacidade e até mesmo a morte (LANA et al., 2014; YI; LANGFORD, 2006). Pesquisas, como as de Behm (2005) e Manuele (2013), mostram que arquitetos e engenheiros têm a oportunidade de afetar positivamente a segurança e a saúde dos trabalhadores da construção civil por meio das escolhas que fazem no processo de concepção do empreendimento. Trata-se da "Prevenção através do Projeto", em inglês "Prevention Trough Design" (PtD).

Para Melzner et al. (2013), existe alto potencial de uso do "Building Information Modeling" (BIM) para apoiar e operacionalizar a adoção do PtD, visando proteger os trabalhadores da construção civil por meio da consideração dos requisitos de segurança no processo de projeto. Kamardeen (2010), Zhang et al. (2013), Qi et al. (2014) e Wetzel e Thabet (2015) demonstram a viabilidade e importância do apoio do BIM para atender aos requisitos dos projetos de segurança.

LEÃO, C.B.O.; SERRA, S.M.P. Planejamento da segurança na construção utilizando a modelagem virtual. In: SIMPÓSIO BRASILEIRO DE GESTÃO E ECONOMIA DA CONSTRUÇÃO, 11., 2019, Londrina. Anais [...]. Porto Alegre: ANTAC, 2019. Disponível em: https://www.antaceventos.net.br/index.php/sibragec/sibragec2019/paper/view/470 
Observa-se haver potencial para pesquisas que agreguem a adoção do BIM com a gestão da segurança via PtD. Portanto, nesta pesquisa o projeto é utilizado, na fase planejamento da construção, para simular a programação de medidas de segurança no modelo 4D.

Diante do exposto, o objetivo deste trabalho é identificar pontos de melhoria na integração do planejamento dos sistemas de proteção coletiva (SPC) ao cronograma de físico da obra utilizando a ferramenta PtD (prototipagem virtual) em um modelo BIM 4D. Com isso, espera-se a utilização do PtD se torne oportunidade de mitigação de riscos durante a fase de planejamento da obra. Afinal, conforme destaca Manuele (2008), PtD integra decisões que afetam a segurança e a saúde em todos os estágios do processo de desenvolvimento do empreendimento

\section{REVISÃO DA LITERATURA}

\subsection{Prevenção através do Projeto (PtD)}

Eliminar riscos na fonte pode ser um meio bastante eficaz para lidar com os riscos de acidentes. Talvez a definição mais simples de PtD seja "Construção Segura" ou "Projeto para segurança da construção". Weinstein et al. (2005, p.1125) declararam que "projetar para a segurança da construção implica em abordar a segurança dos trabalhadores da construção na concepção das características permanentes de um empreendimento". Cooke et al. (2008) e Zarges e Giles (2008) argumentam que alguns projetistas não estão preparados para esta abordagem, pois a Saúde e Segurança do Trabalho, tradicionalmente, não foi bem integrada durante a formação de arquitetos ou engenheiros.

Para Ku e Mills (2010), é possível o suporte computacional para o desenvolvimento dos PtD por meio do uso da Prototipagem virtual, integrando planejamento e visualização de planos de construção de projetos de construção.

\subsection{Building Information Modeling (BIM)}

Atualmente, dada a sua abrangência o BIM possui vários domínios $(\mathrm{nD})$ que caracterizam diferentes fases do ciclo de vida de uma edificação, dentre elas: o modelo 3D usado na concepção e o 4D corresponde ao processo de planejamento (KAMARDEEN, 2010).

As ferramentas de modelagem de informações de construção (BIM) oferecem novas abordagens para "projetar" ou minimizar perigos e riscos integrando modelos 3D com procedimentos de construção com o conhecimento da segurança. Acredita-se que o processo de aplicação do BIM possa ser visto, até certo ponto, como uma forma sistemática de gerenciar os riscos (ZOU et al., 2017).

Gambatese, Toole e Behm (2008) afirmam que as pesquisas nesse tema precisam ser aplicadas e apontam meios para medir o impacto e eficácia do PtD. Behm (2008) reitera que o uso do BIM para planejar segurança apresenta vantagens significativas. Com isso, é possível melhorar a performance dos projetos, minimizar perigos e riscos integrando modelos 3D por meio de processos de design iterativo.

\subsection{Sistema de Proteção Coletiva (SPC)}

Para prevenção das quedas em altura, existem três tipos de SPC que são as principais soluções utilizadas atualmente nas obras no Brasil para edifícios.

De acordo com item 18.13.6 da NR-18 (BRASIL, 2018), recomenda-se que em todo perímetro da construção de edifícios com mais de quatro pavimentos ou altura equivalente, seja obrigatória a instalação de uma plataforma principal de proteção na 


\section{SIBRAGEC - ELAGEC 2019 - de 23 a 25 de Outubro - LONDRINA - PR}

altura do primeiro pavimento que esteja, no mínimo, um pé-direito acima do nível do terreno. Essa plataforma deve ter, no mínimo, 2,50m de projeção horizontal da face externa da construção e um complemento de $0,80 \mathrm{~m}$ de extensão, com inclinação de $45^{\circ}$, a partir de sua extremidade.

No que se refere à plataforma secundária, o item 18.13.7 da NR-18 (BRASIL, 2018), recomenda como medida alternativa ao seu uso, a instalação de Sistema Limitador de Quedas de Altura (SLQA) com a utilização de redes de segurança do sistema "V".

Conforme o item 18.13.12.6 da NR-18 (BRASIL, 2018), na parte inferior do SLQA, a rede deve permanecer o mais próximo possível do plano de trabalho. No projeto analisado a rede foi fixada dois pavimentos abaixo do plano de trabalho. Essa solução é justificada pelo mesmo item da NR-18 o qual recomenda que entre a parte inferior do SLQA e a superfície de trabalho deve ser observada uma altura máxima de seis metros.

\section{MÉTODO}

Esta pesquisa é classificada como exploratória e descritiva. A abordagem proposta para a integração do planejamento de estruturas temporárias com o processo de construção de um edifício, consistiu nas seguintes etapas:

1. Definição do projeto dos sistemas de proteção coletiva (SPC);

2. Representação dos componentes do SPC por meio de representação BIM-3D;

3. Inclusão dessas estruturas temporárias no modelo BIM-3D;

4. Planejamento da inclusão das atividades relacionadas a montagem e desmontagem dessas estruturas, para simulação e visualização das etapas da obra no modelo BIM-4D;

5. Identificação de oportunidades de melhoria ou deficiências na programação, especialmente no que se refere a segurança em obra.

Os projetos foram desenvolvidos em plataformas computacionais BIM-Revit 2015. O estudo no modelo BIM-3D foi baseado em projeto real de uma edificação escolar de, aproximadamente, $8.400 \mathrm{~m}^{2}$, distribuídos em 7 pavimentos. Para tanto, foram utilizados o projeto arquitetônico e o projeto de estruturas temporárias dos SPC. O software Navisworks permitiu a consideração do planejamento na modelagem 4D.

\section{RESULTADOS E DISCUSSÕES}

\subsection{Estruturas temporárias para proteção coletiva}

No projeto em questão era prevista a utilização de três tipos de SPC: Plataforma primária, também chamada de Bandeja; Sistema Limitador de Quedas de Altura (SLQA) e proteção de periferia tipo Guarda-corpo-Rodapé (GcR).

A Figura 1 mostra a representação da plataforma no projeto BIM-4D.

Para obra em questão, foi prevista a utilização de SLQA, conforme Figura 2.

Na periferia da edificação, foi prevista a instalação de GcR, com altura de 1,20m (um metro e vinte centímetros) para o travessão superior e $0,70 \mathrm{~m}$ (setenta centímetros) para o travessão intermediário, rodapé com altura de $0,20 \mathrm{~m}$ (vinte centímetros) e os vãos entre travessas preenchidos com tela ou outro dispositivo que garanta o fechamento seguro da abertura. A Figura 3 mostra a representação do GcR adotada nesta pesquisa. Estes 
SIBRAGEC - ELAGEC 2019 - de 23 a 25 de Outubro - LONDRINA - PR

dispositivos são instalados apenas entre o plano de trabalho e a parte inferior do SLQA, uma vez que os pavimentos abaixo do sistema limitador estão com alvenaria de periferia finalizada.

\section{Figura 1 - Plataforma primária}

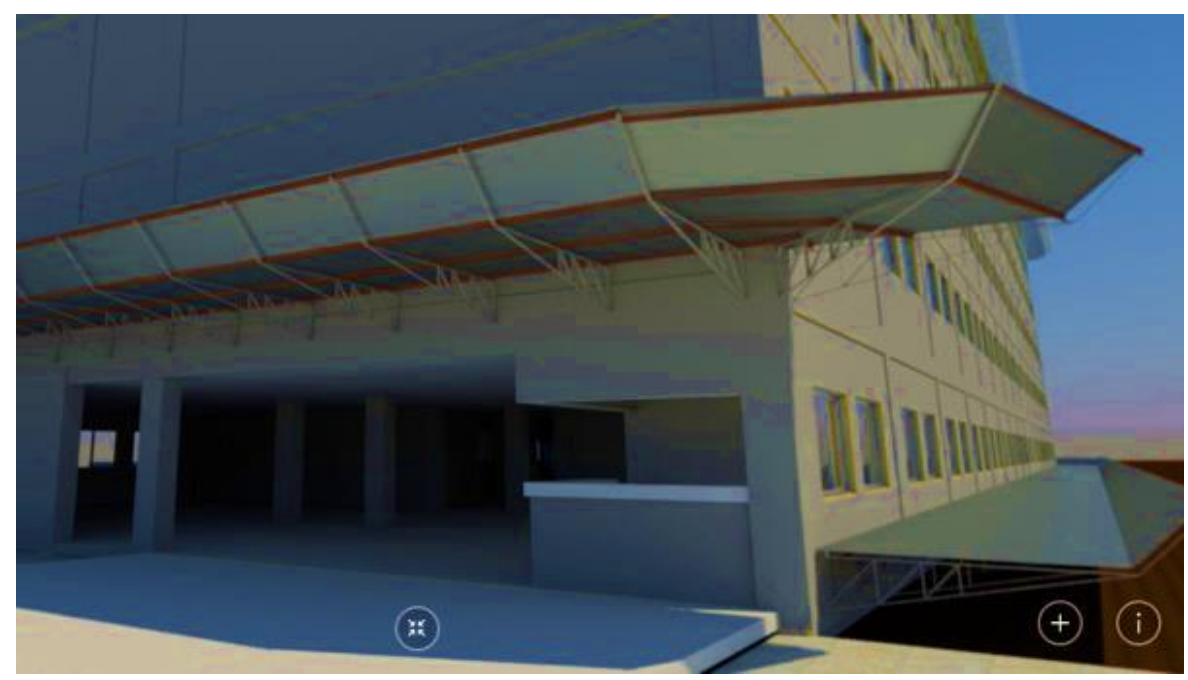

Figura 2 - Sistema LQA adotados no modelo BIM-4D

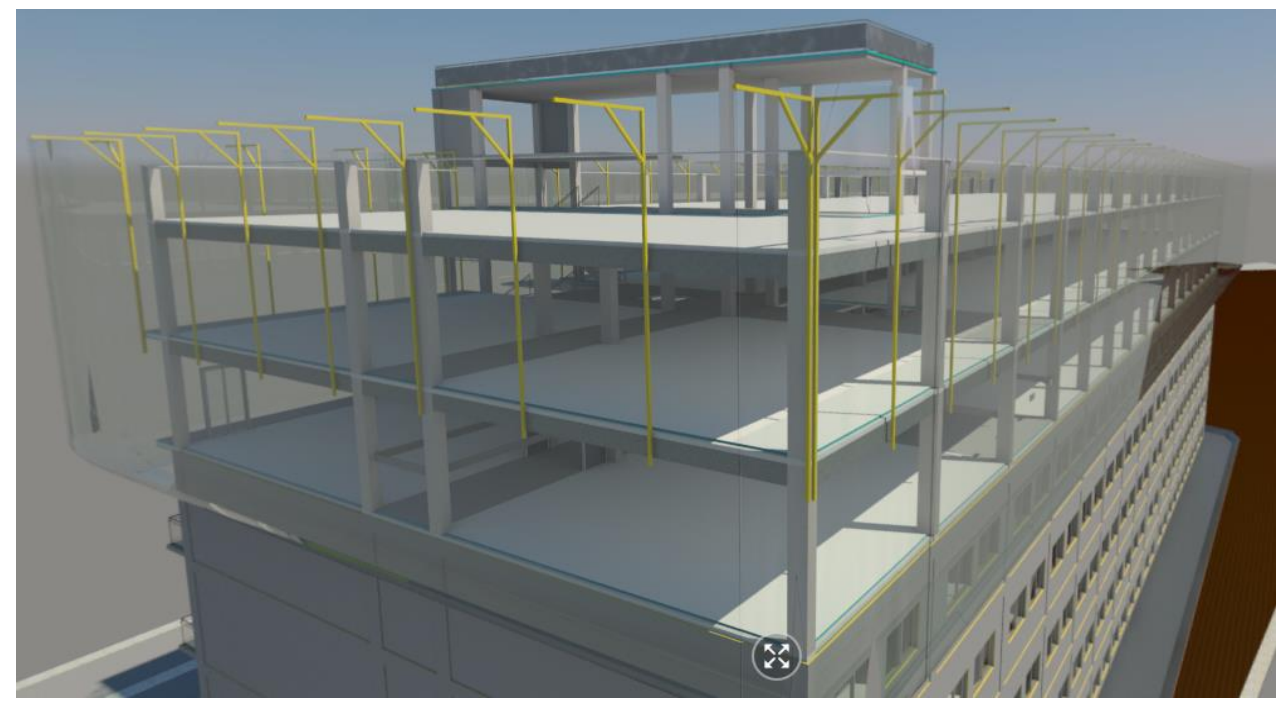




\section{Figura 3 - Exemplo de GcR}
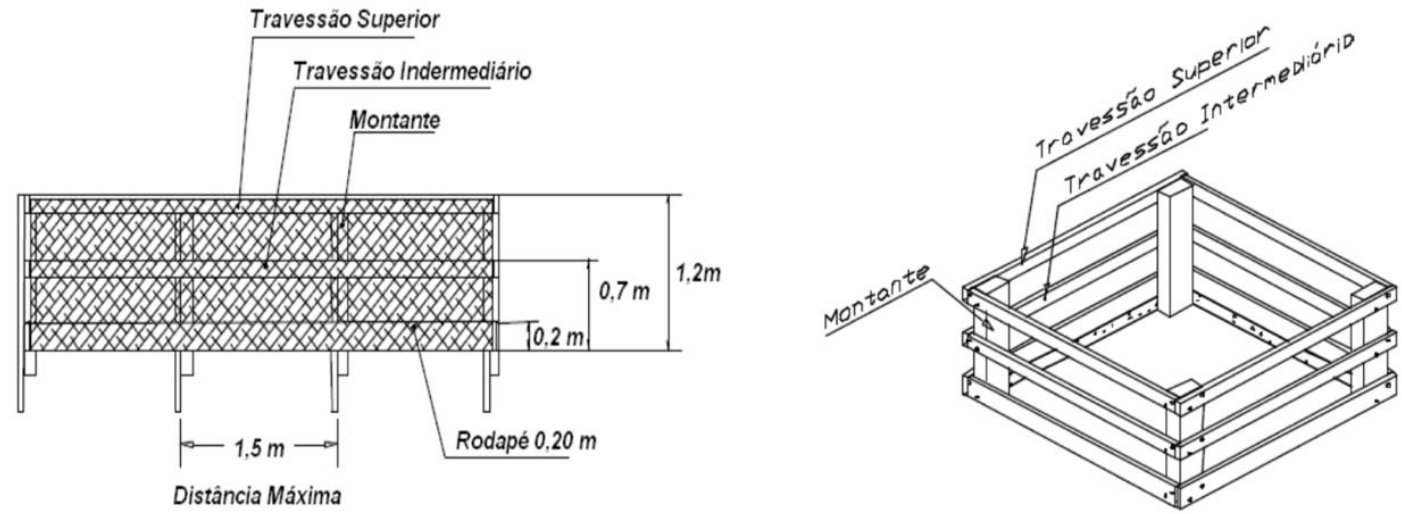

Fonte: Fundacentro (2003)

\subsection{Modelagem das estruturas temporárias}

Para inclusão dos SPC ao modelo BIM-3D, houve dificuldade devido à falta de arquivos de famílias ou bibliotecas dos fornecedores para alimentação dos modelos virtuais. Para atender essa demanda foi necessário realizar modelagem dos componentes que não estavam disponíveis previamente. No entanto, a falta de precisão em informações relacionadas aos atributos dessas estruturas dificultou o processo.

As informações faltantes precisaram ser determinadas de acordo com normas e experiência dos pesquisadores, buscando seguir a recomendação de Toole e Gambatese (2008), que sugerem que sejam escolhidos os materiais e sistemas que são inerentemente mais seguros, de acordo com a engenharia da construção.

Logo na primeira etapa da pesquisa foi possível verificar benefícios da prototipagem virtual ao antever inconsistências de projeto, conforme mencionado por Ku e Mils (2010). Essas foram resolvidas para evitar que os trabalhadores sejam expostos ao risco.

\subsection{Planejamento, simulação e visualização das etapas da obra}

Com as estruturas temporárias inclusas no modelo BIM-3D, foram definidas atividades a cada um dos elementos destas estruturas. A obra possuía um cronograma físico, portanto buscou-se incluir as atividades relacionadas à montagem e desmontagem dos SPC, respeitando o plano de ataque definido. As premissas adotadas no planejamento foram:

\subsubsection{Plataforma principal}

- Instalada logo após a concretagem do primeiro pavimento;

- Retirada quando terminado o revestimento externo da estrutura.

A Figura 4 mostra o momento de instalação da plataforma primária no primeiro pavimento, conforme cronograma físico. 
SIBRAGEC - ELAGEC 2019 - de 23 a 25 de Outubro - LONDRINA - PR

Figura 4 - Simulação da montagem da Plataforma principal

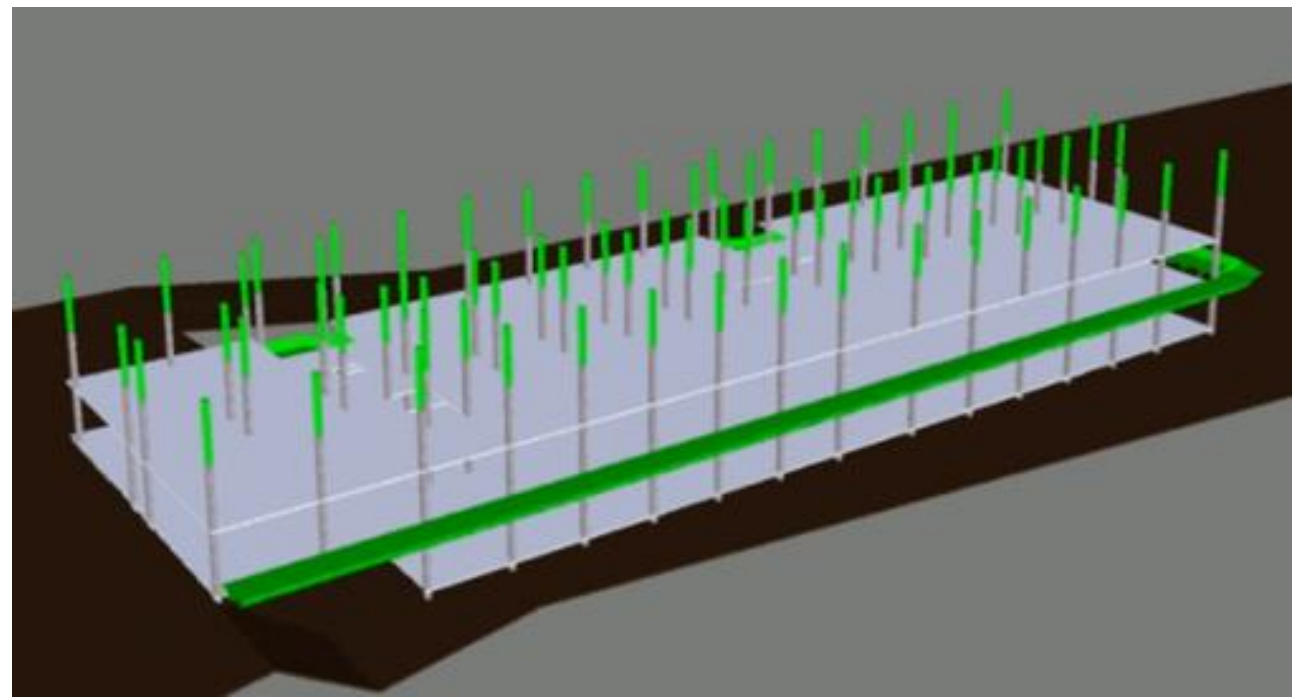

\subsubsection{Redes de segurança SLQA}

- Instalada durante a execução do terceiro pavimento;

- Durante a execução do terceiro pavimento, o escoramento do primeiro pavimento é parcialmente removido;

- A alvenaria inicia após retirada parcial do escoramento do pavimento.

- O quarto pavimento só é liberado para ser iniciado, após a conclusão da alvenaria do primeiro pavimento, pois a rede só pode ser movimentada se o perímetro do primeiro pavimento estiver protegido;

- A movimentação da rede (subida) acontece quando o segundo pavimento estiver protegido.

A Figura 5 mostra o momento em o SLQA é instalado.

\section{Figura 5 - Simulação da montagem do SLQA}

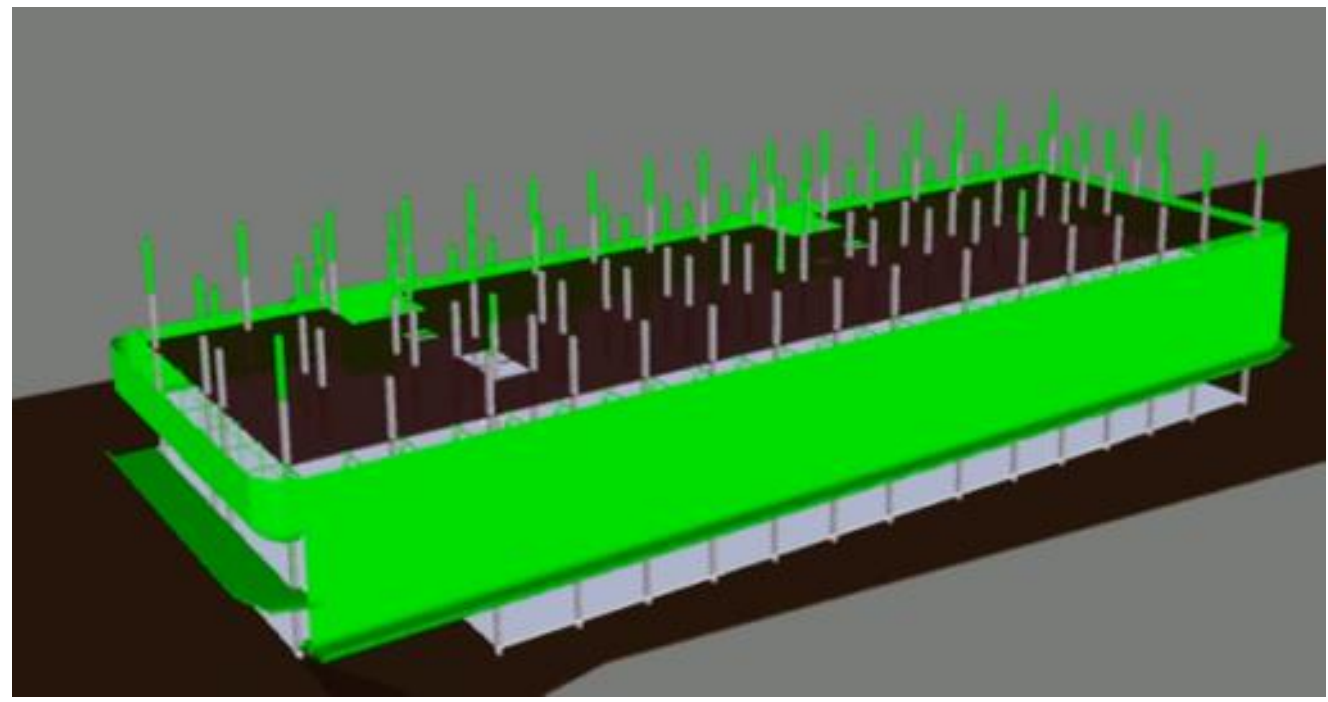


SIBRAGEC - ELAGEC 2019 - de 23 a 25 de Outubro - LONDRINA - PR

A Figura 6 mostra dois equipamentos modelados incorporados ao edifício. Quando finalizada a alvenaria no edifício, será feita a desmontagem do SLQA.

\section{Figura 6 - Vista dos SPC incorporados ao modelo BIM-4D}

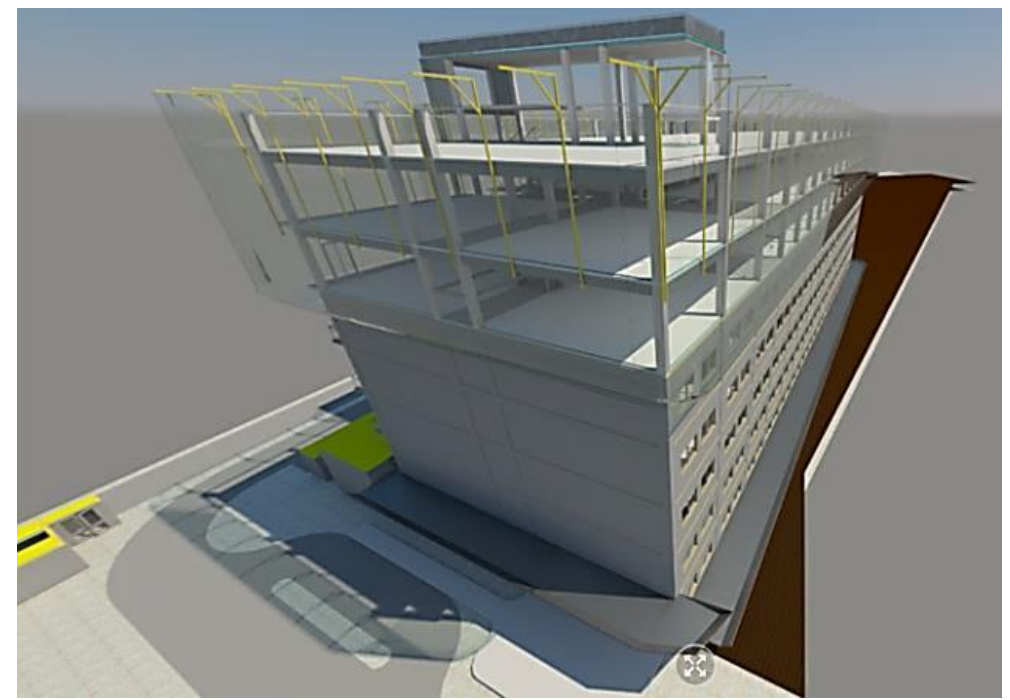

A construtora explicou que a montagem e desmontagem dos GcR demandam muito tempo de exposição dos trabalhadores ao perímetro do pavimento, obrigando os trabalhadores a se exporem ao risco. Por isso, buscam reduzir a necessidade de realizar essa atividade. Apesar dessa decisão poder reduzir o ritmo de trabalho, por outro lado garantiu que o risco de queda em altura será reduzido, uma vez que a rede será movimentada apenas quando o pavimento estiver fechado.

A transferência do SLQA para o pavimento seguinte pode ser considerada uma atividade arriscada. Pois, são estruturas pesadas movimentadas manualmente, com auxílio de um pequeno guincho. Com o intuito de reduzir o risco nessa atividade, o ideal é que seja realizada por equipe capacitada com ferramentas adequadas.

Com base nessas premissas, todas as atividades foram planejadas no modelo BIM-4D. A Figura 7 ilustra a simulação da sequência construtiva incluindo as atividades relacionadas com os SPC. 
SIBRAGEC - ELAGEC 2019 - de 23 a 25 de Outubro - LONDRINA - PR

Figura 7 - Modelo 4D - Programação das atividades

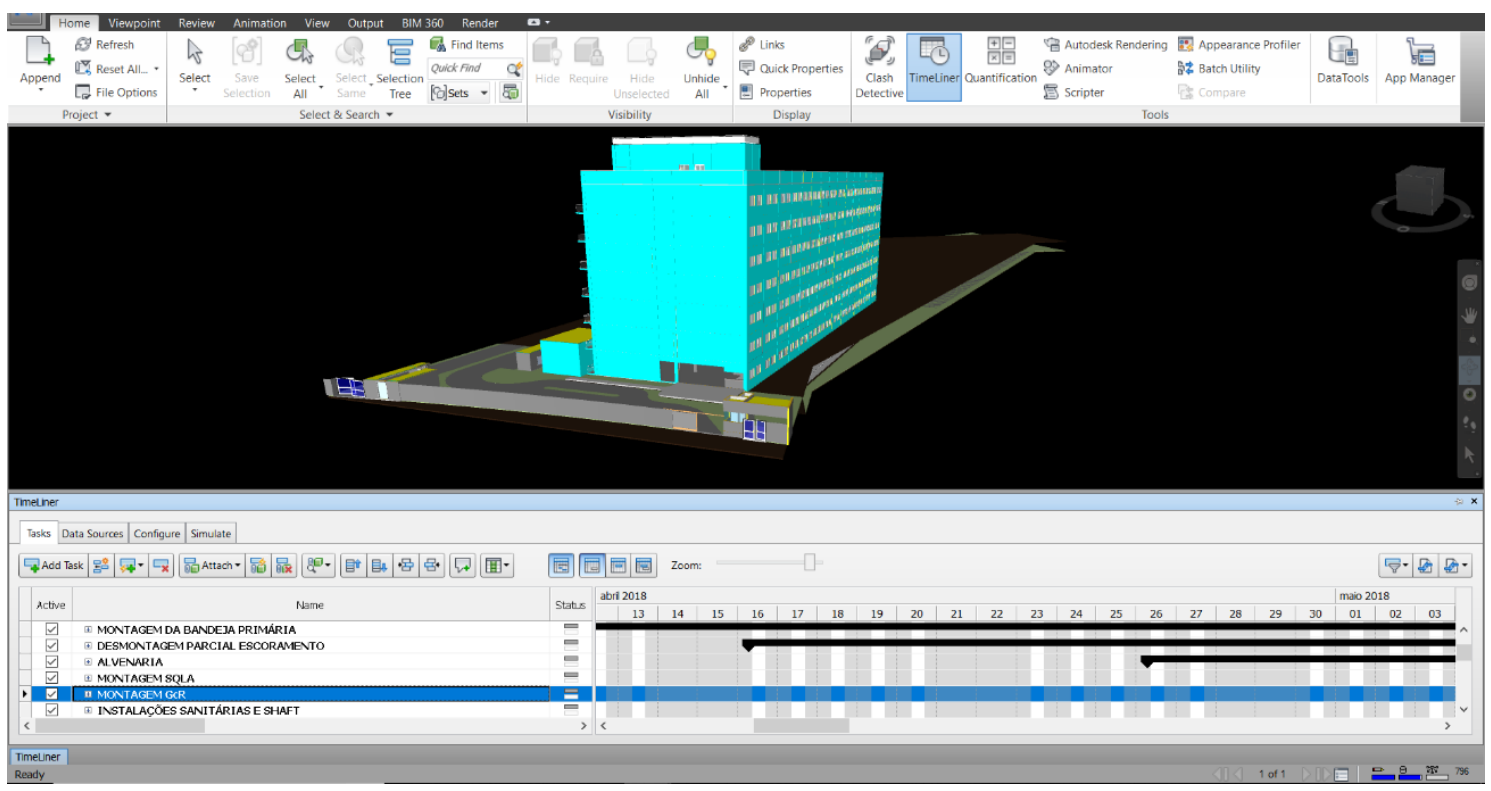

\subsection{Oportunidades de melhoria na programação}

Antes da elaboração deste cronograma, a construtora não possuía registros sobre a sequência e programação de montagem e desmontagem dos SPC citados neste estudo. A inclusão das atividades no cronograma facilitou o registro e o controle delas. Por exemplo, após a concretagem do primeiro pavimento, os serviços de montagem de forma para o segundo pavimento continuariam, sem que tivesse nenhuma predecessora relacionada aos SPC.

Com a integração das atividades relacionadas a montagem e desmontagem dos SPC, outra atividade não deve ser iniciada, antes que a bandeja primária seja instalada. A inserção dos SPC como predecessora pode ajudar a reduzir o risco de acidentes.

A prototipagem virtual, além de auxiliar na detecção de inconsistências no projeto, possibilita a identificação de deficiências na programação. Na Figura 8, observa-se a falha no agendamento da montagem do GcR que foi registrada durante as primeiras etapas da modelagem 4D, que foi corrigida posteriormente. Na imagem, percebe-se dois pavimentos concretados e sem a presença de GcR. Essa visualização, por meio da simulação 3D, facilita a detecção de falhas como essa. 
SIBRAGEC - ELAGEC 2019 - de 23 a 25 de Outubro - LONDRINA - PR

\section{Figura 8 - Ausência de GcR na etapa de concretagem do pavimento}

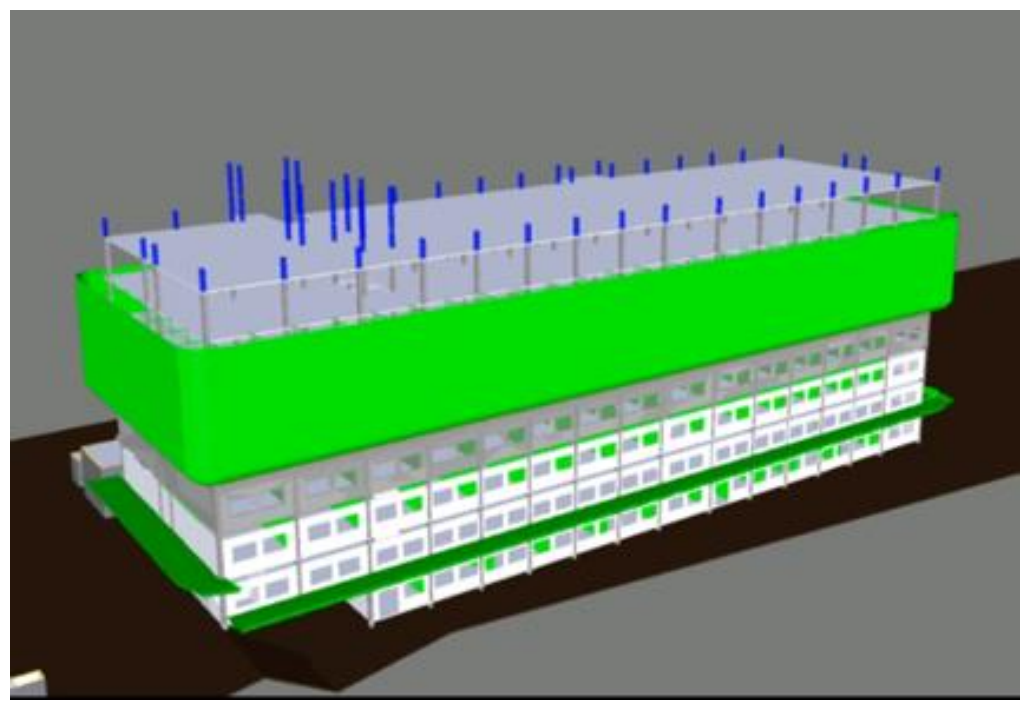

\section{CONSIDERAÇÕES FINAIS}

O objetivo do artigo foi identificar pontos de melhoria na integração do planejamento dos sistemas de proteção coletiva (SPC) ao cronograma de físico da obra utilizando a ferramenta PtD (prototipagem virtual) em um modelo BIM-4D. O uso da ferramenta PtD (prototipagem virtual) integrada com BIM-4D mostrou-se como um possível instrumento para prevenção dos riscos de acidentes, na etapa de planejamento da obra.

Por meio do modelo, foi possível simular e visualizar a obra, de modo que o construtor possa antever o risco das atividades e planejar com antecedência as alocações de recursos de segurança.

Ao longo da continuidade da pesquisa poderão ser apresentadas diretrizes para melhoria dos projetos de SPC, de forma a retroalimentar o processo de concepção do empreendimento.

\section{REFERÊNCIAS}

BEHM, M. Linking construction fatalities to the design for construction safety concept. Safety Science, 2005, v.43, n.8, p.589-611.

BEHM, M. Construction Sector. Journal of Safety Research, 2008, v.39, n.2, p.175-178.

COOKE, T.; LINGARD, H.; BLISMAS, N.; STRANIERI, A. ToolSHeDTM: the development and evaluation of a decision support tool for health and safety in construction design.

Engineering, Construction and Architectural Management, 2008, v.15, n.4, p.336-351.

FUNDACENTRO (2003). RTP 01-Medidas de Proteção Contra Quedas de Altura. São Paulo: Fundacentro. http://www.fundacentro.gov.br/biblioteca/recomendacao-tecnica-deprocedimento/download/Publicacao/147/rtp01-pdf.

GAMBATESE, J.; TOOLE, T.M.; BEHM, M. Prevention through Design Practice and Research: A Construction Industry Perspective. In: Proceedings of CIB W99 International Conference 14th Rinker International Conference, March, 2008.

KAMARDEEN, I. 8D BIM: Modelling tool for accident prevention through design. Faculty of Build Environment, University of New South Wales, Australia, 2010. 


\section{SIBRAGEC - ELAGEC 2019 - de 23 a 25 de Outubro - LONDRINA - PR}

KU, K.; MILLS, T. Research needs for Building Information Modeling for Construction Safety. In: Proceedings of the 44th ASC National Conference, April 2-5, 2010. Auburn, AL, USA.

LANA, L. D.; QUADROS, J.N.; WEISE, A.D.; REIS, R.P.; ROSA, L.C.; BULIGON, S.M. Avaliação dos riscos do trabalho em altura na construção civil. Revista Produção Online, 2014, v.14, n.1, p. 344-363.

MANUELE, F. Prevention through Design (PtD): History and future. Journal of Safety Research, v.39, 2008, p. 127-130.

MANUELE, F. On the practice of Safety, 2013. 4th Ed., Hoboken, NJ: Wiley.

MELZNER, J.; ZHANG, S.; TEIZER, J.; BARGSTÄDT, H.J. A case study on automated safety compliance checking to assist fall protection design and planning in building information models. Construction Management and Economics, 2013, v.31, n.6, p.66-674.

QI, J.; ISSA, R.R.A.; OLBINA, S.; HINZE, J. Use of Building Information Modeling in Design to Prevent Construction Worker Falls. Journal of Computing in Civil Engineering, 2014, v.28, n.5, p.1-10.

TOOLE, T. M.; GAMBATESE, J. The trajectories of prevention through design in construction. Journal of Safety Research, 2008, v.39, n.2, p.225-230.

WEINSTEIN, M.; GAMBATESE, J.; HECKER, S. Can Design Improve Construction Safety?: assessing the impact of a collaborative safety-in-design process. Journal of Construction

Engineering and Management, 2005, v.131, n.10.

WETZEL, E.M; THABET, W.Y. The use of a BIM-based framework to support safe facility management processes. Automation in Construction, 2015, v.60, p.12-24.

YI, K.J.; LANGFORD, D. Scheduling-based risk estimation and safety planning for construction projects. Journal of Construction Engineering and Management, 2006, v.132, n.6, p.626-635.

ZHANG, S.; TEIZER, J.; LEE, J.K..; EASTMAN, C.M.; VENUGOPAL, M. Building Information Modeling (BIM) and Safety: Automatic Safety Checking of Construction Models and Schedules. Automation in Construction, 2013, v.29, p.183-195.

ZARGES, T.; GILES, B. Prevention through Design (PtD). Journal of Safety Research, 2008. v.39, n.2, p.123-126.

ZOU, Y., KIVINIEMI, A.; JONES, S.W. A review of risk management through BIM and BIMrelated technologies. Safety Science, 2017, v.97, p.88-98.

\section{AGRADECIMENTOS}

Agradecemos o apoio da Coordenação de Aperfeiçoamento de Pessoal de Nível Superior (CAPES) concedido por meio de bolsa de estudo, da Fundação de Amparo à Pesquisa do Estado de São Paulo (FAPESP), assim como à empresa colaboradora com a pesquisa. 